\title{
Characteristics and prognosis of telangiectatic osteosarcoma: a population-based study using the Surveillance, Epidemiology and End Results (SEER) database
}

\author{
Mingfang Xu ${ }^{1 \#}$, Nan Dai ${ }^{1 \#}$, Xueqin Yang ${ }^{1}$, Wei Guan ${ }^{1}$, Yu Pu ${ }^{1}$, Dong Wang ${ }^{1}$, Liangjun Yin ${ }^{2}$, Mao Nie \\ ${ }^{1}$ Cancer Center of Daping Hospital, Army Medical University, Chongqing, China; ${ }^{2}$ Department of Orthopaedic Surgery, The Second Affiliated \\ Hospital of Chongqing Medical University, Chongqing, China \\ Contributions: (I) Conception and design: M Xu, M Nie; (II) Administrative support: X Yang, D Wang, L Yin; (III) Provision of study materials or \\ patients: M Xu; (IV) Collection and assembly of data: M Xu, N Dai; (V) Data analysis and interpretation: M Xu, N Dai; (VI) Manuscript writing: All \\ authors; (VII) Final approval of manuscript: All authors. \\ \#These authors contributed equally to this work. \\ Correspondence to: Mao Nie, PhD, MD. Department of Orthopaedic Surgery, The Second Affiliated Hospital of Chongqing Medical University, 76 \\ Linjiang Road, Yuzhong District, Chongqing, China. Email: 302218@cqmu.edu.cn.
}

Background: Telangiectatic osteosarcoma (TOS) is a rare type of osteosarcoma for which limited clinical data is available. Furthermore, the clinical characteristics and prognosis of TOS remain unclear.

Methods: A large population-based cohort analysis was conducted using the Surveillance, Epidemiology and End Results (SEER) registry. The data of TOS and conventional osteosarcoma (COS) patients from 2000 to 2017 were collected. The categorical variables were assessed by Chi-squared tests. Kaplan-Meier curves and log-rank (Mantel-Cox) tests were used to examine the survival outcomes between the groups. Cox proportional hazard models were used for univariate and multivariate analyses of TOS patient survivalrelated variables.

Results: A total of 141 TOS patients and 2961 COS patients were included in this analysis, and the mean age at diagnosis was 23.5 and 29.4 years, respectively. Compared to COS patients, TOS patients were more likely to be under 20 years old $(61.7 \%$ vs. $51.7 \%, \mathrm{P}=0.022)$, and without a second peak of incidence after 60 years of age. The median overall survival (mOS) of TOS patients was not reached compared to a median survival of 84 months for COS patients (hazard ratio $0.75,95 \%$ confidence interval 0.59 to $0.95, \mathrm{P}=0.0175$ ). After adjusting these data for age at diagnosis, stage, and surgery at the primary site, no significant differences in mOS were observed between the two groups. In univariate analyses, being under 20 years of age, having localized or regional stage disease, and having undergone surgery were associated with a decreased risk of death. Subsequent multivariate analysis indicated that age at diagnosis, stage, and surgery at the primary site were all independent predictors of prognosis in TOS patients.

Conclusions: Patients with TOS were younger than patients with COS and did not show a second peak after 60 years of age. Age, summary stage at diagnosis, and surgery at the primary site were independent predictors of survival for TOS patients.

Keywords: Telangiectatic osteosarcoma (TOS); clinical characteristics; SEER; prognosis; median overall survival (mOS)

Submitted Nov 18, 2020. Accepted for publication Jan 14, 2021.

doi: 10.21037/atm-20-8001

View this article at: http://dx.doi.org/10.21037/atm-20-8001 


\section{Introduction}

Osteosarcoma is among the most prevalent forms of non-hematopoietic primary malignant bone cancer (1). Approximately $90 \%$ of osteosarcomas are of the common types including osteoblastoma, fibroblastoma, and chondroblastoma. Numerous independent osteosarcoma histological types that have been differentiated from conventional osteosarcomas (COS) based upon their behaviors and characteristics upon $\mathrm{X}$-ray analysis, including telangiectatic osteosarcoma (TOS), small cell osteosarcoma, intraosseous well-differentiated osteosarcoma, high-grade surface osteosarcoma, periosteal osteosarcoma, parosteal osteosarcoma, osteosarcoma in Paget's disease of the bone, and central osteosarcoma (2-5).

TOS is a rare osteosarcoma subtype that accounts for less than $4 \%$ of all cases (6), and was first described in 1854 by Paget. There were no significant differences in common symptoms and signs between TOS and COS, including recent local pain, soft tissue mass, or both. TOS consists of multiple characteristic dilated aneurysmal cavities containing blood, with highly malignant sarcoma cells being present at the peripheral margins and septations surrounding these cavities (7). As this is a very rare form of cancer, no largescale clinical studies of TOS have been conducted to date, and case reports (8-10) and animal studies (11-13) thus account for the bulk of research related to this condition. Given these limitations, the clinical characteristics and prognosis of TOS remain poorly understood. Previously, TOS was presumed to be associated with a poorer prognosis compared to COS (14). However, some researchers have found TOS to be associated with no significant survival differences or with mildly improved survival as compared to patients with conventional high-grade intramedullary osteosarcoma $(15,16)$.

Owing to the rarity of this condition and the discrepancies in the pertinent prognostic data available, the present study evaluated the clinical characteristics and prognosis of TOS using the Surveillance, Epidemiology, and End Result (SEER) database maintained by the National Cancer Institute.

We present the following article in accordance with the STROBE reporting checklist (available at http://dx.doi. org/10.21037/atm-20-8001).

\section{Methods}

\section{Study population}

The SEER database provides clinical information on many tumors, which is very useful for clinical analysis of rare tumors (17-19). Through the SEER database (http://seer. cancer.gov/) all patients from 2000-2017 who had been diagnosed with TOS (IDC, 9183/3) were identified. Patients diagnosed with osteosarcoma that was not otherwise specified (NOS; IDC, 9180/3), were defined as COS patients in this present study, based upon the ICD-0-3/WHO 2008 criteria. The SEER*Stat 8.3.6.1 software platform was used for case identification. A total of 145 TOS and 3,115 COS patients were identified for this study. Patients were excluded from this analysis if they: (I) lacked a pathologically confirmed diagnosis; (II) were diagnosed upon death or via autopsy; (III) had uncertain overall survival outcomes; or (IV) had a history of other tumors. Based on these criteria, a final cohort of 141 TOS patients and 2,961 COS patients were included in this present analysis.

\section{Statistical analysis}

Categorical data were presented as numbers with percentages and was compared via chi-squared tests, while continuous data were presented as means or medians with corresponding ranges. Patient median overall survival (mOS) was measured as the time between diagnosis and the most recent follow-up or all-cause mortality. Survival outcomes were compared between groups using Kaplan-Meier curves and log-rank (Mantel-Cox) tests. In models assessing patient survival outcomes, age at diagnosis, gender, race, year of diagnosis, grade, primary site (laterality), summary stage, and surgery at the primary site were included as variables. Cox proportional hazard models were used for univariate and multivariate analyses of TOS patient survivalrelated variables. A two-sided $\mathrm{P}$ value $<0.05$ was used as the significance threshold in this analysis, and SPSS v23 (IBM Corp, NY, USA) was used for all statistical analyses.

\section{Ethics approval}

The study was conducted in accordance with the Declaration of Helsinki (as revised in 2013). As cancer is considered a reportable disease in the United States, no patient consent was necessary for the use of the SEER database.

\section{Results}

\section{Patient characteristics}

By reviewing the SEER database, we identified 4,630 patients who had been diagnosed with osteosarcoma between 
A

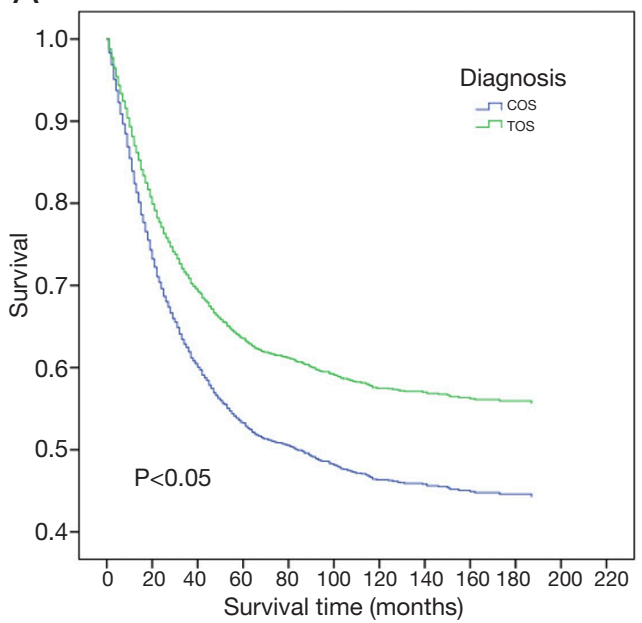

B

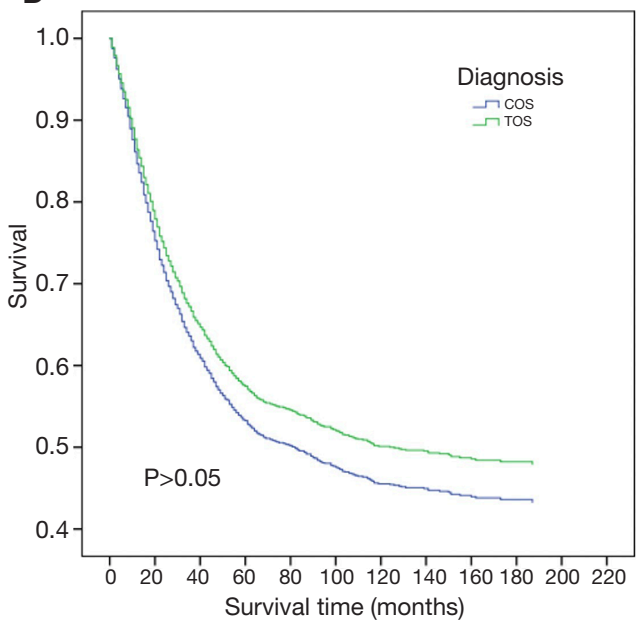

Figure 1 Kaplan-Meier survival curves of COS and TOS patients. (A) Kaplan-Meier curves indicated that TOS patients survived significantly longer than COS patients (not reached vs. 84 months; HR 0.75, 95\% CI: 0.59 to 0.95, P=0.0175); (B) Kaplan-Meier curves indicated that the mOS of TOS patients did not differ significantly from that of COS patients after adjusting for age at diagnosis, stage, and surgery at primary site. TOS, telangiectatic osteosarcoma; HR, hazard ratio; CI, confidence interval; COS, conventional osteosarcoma; mOS, median overall survival.

2000 and 2017, including 3,115 COS patients and 145 TOS patients. TOS patients accounted for just $3.1 \%$ of the total osteosarcoma cases. Based upon the exclusion criteria, 2,961 COS patients and 141 TOS patients were finally included in the present study. These TOS and COS patients had a mean age at diagnosis of 23.5 and 29.4 years, respectively. Overall clinical and demographic data pertaining to these two patient cohorts have been compiled in Table 1.

\section{Survival analysis}

Significant differences were detected between the COS and TOS patient cohorts with respect to age at diagnosis, surgery at the primary site, and mOS. However, after these data were adjusted for age at diagnosis, tumor stage, and surgery at the primary site, no significant differences in mOS were observed between these groups. In addition, no significant differences were detected between these two patient cohorts with respect to patient gender, race, year of diagnosis, grade, laterality, or summary stage.

The survival of TOS and COS patients were compared (Figure 1A). The mOS of TOS patients was not reached as more than $50 \%$ of these patients are still alive (range, 1-209 months), while the mOS of COS patients was 84 months (range, 1-215 months). There was a significant difference between these patient cohorts, with hazard ratio (HR) $0.75,95 \%$ confidence interval (CI) 0.59 to 0.95 , and $\mathrm{P}=0.0175$. However, no significant differences in mOS were detected between the two groups after adjusting for age at diagnosis, stage, and surgery at the primary site (Figure 1B).

The survival outcomes of TOS patients as a function of age at diagnosis were examined (Figure 2). Kaplan-Meier curves revealed that patients less than 20 years old at the time of diagnosis had significantly better survival outcomes compared to other patients (not reached $v s .49$ months, HR $0.32,95 \%$ CI: 0.18 to $0.58, \mathrm{P}<0.0001)$, with patients less than 10 years old showing the best prognosis. TOS patient survival was also compared as a function of patient gender, race, grade, and laterality, but none of these factors were associated with survival $(\mathrm{P}>0.05)$ (Figure 3). In addition, TOS patient survival was compared as a function of summary stage and surgery of the primary site (Figure 4). Patients with localized or regional disease had significantly better survival outcomes compared to patients with advanced disease (not reached vs. 29.5 months, HR 0.13, 95\% CI: 0.06 to $0.29, \mathrm{P}<0.0001)$. Similarly, patients who underwent surgical tumor resection showed significantly improved survival compared to patients who did not undergo surgery (not reached vs. 100 months, HR 0.27, 
Table 1 Demographic and clinic charaWcteristics of patients diagnosed with TOS and COS between 2000 and 2017 in the SEER database

\begin{tabular}{|c|c|c|c|}
\hline Diagnosis & TOS (\%) & $\cos (\%)$ & $P$ value \\
\hline $\mathrm{N}$ & 141 & 2,961 & \\
\hline Age at diagnosis & & & 0.022 \\
\hline$<20$ & $87(61.7)$ & $1,532(51.7)$ & \\
\hline 20-39 & $30(21.3)$ & $571(19.3)$ & \\
\hline $40-59$ & $13(9.2)$ & $435(14.7)$ & \\
\hline$\geq 60$ & $11(7.8)$ & $423(14.3)$ & \\
\hline Race & & & 0.496 \\
\hline White & $111(78.7)$ & $2,218(74.9)$ & \\
\hline Black & $20(14.2)$ & 452 (15.3) & \\
\hline Other & $10(7.1)$ & $291(9.8)$ & \\
\hline Gender & & & 0.219 \\
\hline Female & $56(39.7)$ & $1,332(45.0)$ & \\
\hline Male & $85(60.3)$ & $1,629(55.0)$ & \\
\hline Year of diagnosis & & & 0.551 \\
\hline 2000-2004 & $43(30.5)$ & $793(26.8)$ & \\
\hline 2005-2009 & $43(30.5)$ & $832(28.1)$ & \\
\hline 2010-2014 & $34(24.1)$ & $812(27.4)$ & \\
\hline 2015-2017 & $21(14.9)$ & $524(17.7)$ & \\
\hline Laterality & & & 0.001 \\
\hline Left & $78(55.3)$ & $1263(42.7)$ & \\
\hline Right & $56(39.7)$ & $1,238(41.8)$ & \\
\hline Other & $7(5.0)$ & $460(15.5)$ & \\
\hline Grade & & & 0.134 \\
\hline I-II & $3(2.1)$ & $185(6.2)$ & \\
\hline III-IV & $99(70.2)$ & $1,983(67.0)$ & \\
\hline Unknown & $39(27.7)$ & $793(26.8)$ & \\
\hline Stage & & & 0.155 \\
\hline Localized & $49(34.8)$ & $811(27.4)$ & \\
\hline Regional & $51(36.2)$ & $1,046(35.3)$ & \\
\hline Distant & $24(17.0)$ & $611(20.6)$ & \\
\hline Unknown & $17(12.1)$ & $493(16.6)$ & \\
\hline Status & & & 0.053 \\
\hline Alive & $88(62.4)$ & $1,602(54.1)$ & \\
\hline Deceased & $53(37.6)$ & $1,359(45.9)$ & \\
\hline
\end{tabular}

Table 1 (continued)
Table 1 (continued)

\begin{tabular}{llll}
\hline Diagnosis & TOS (\%) & COS (\%) & P value \\
\hline T staging & & & 0.182 \\
T1 & $25(17.7)$ & $430(14.5)$ & \\
T2 & $31(22.0)$ & $511(17.3)$ & \\
T3 & $3(2.1)$ & $39(1.3)$ & \\
Unknown & $82(66.5)$ & $1,981(66.9)$ & \\
N staging & & & \\
N0 & $62(44.0)$ & $1,092(36.9)$ & \\
N1 & $2(1.4)$ & $26(0.9)$ & \\
Unknown & $77(54.6)$ & $1,843(62.2)$ & \\
M staging & & & \\
M0 & $58(41.1)$ & $967(32.7)$ & \\
M1 & $7(5.0)$ & $243(8.2)$ & \\
Unknown & $76(53.9)$ & $1,751(59.1)$ & \\
Surgery & & & \\
No & $15(10.6)$ & $549(18.5)$ & \\
Yes & $126(89.4)$ & $2,412(81.5)$ & \\
\hline
\end{tabular}

TOS, telangiectatic osteosarcoma; COS, conventional osteosarcoma.

95\% CI: 0.09 to $0.79, \mathrm{P}<0.05)$.

The overall 5 - and 10 -year survival rates of TOS patients were $48.2 \%(n=68)$ and $20.6 \%(n=29)$, respectively. Patients who were diagnosed at a younger age had better outcomes, with patients less than 20 years-old showing a 5 -year survival rate of $59.8 \%$, compared to rates of just $36.7 \%, 30.8 \%$, and $9.1 \%$ in patients aged $20-39,40-59$, and greater than 60 years old, respectively. However, there were no significant differences in the 10 -year survival among patients aged less than 20,20-39, and 40-59 years (Table 2).

Lastly, univariate and multivariate analyses were conducted to evaluate the relationships between key clinical variables and patient mOS. In univariate analyses, an age of less than 20 years old was associated with a better prognosis. Patients who had localized or regional disease and patients who underwent surgery also showed better survival outcomes in univariate analysis (HR 0.41; 95\% CI: 0.19 to $0.88 ; \mathrm{P}<0.05)$. Tumor grade, however, was not significantly associated with patient survival (Table 3). Age, summary stage, and surgery at the primary site were included as 

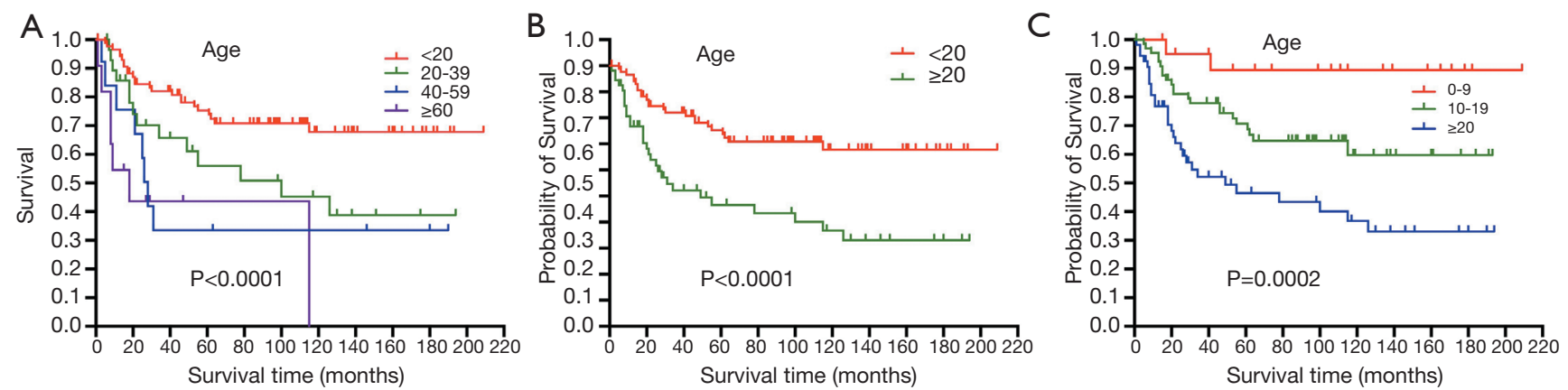

Figure 2 Kaplan-Meier curves revealed that TOS patients less than 20 years old survived significantly longer than other patients (not reached vs. 49 months, HR 0.32, 95\% CI: 0.18 to $0.58, \mathrm{P}<0.0001$ ), with patients less than 10 years old exhibiting the best prognosis. TOS, telangiectatic osteosarcoma; HR, hazard ratio; CI, confidence interval.
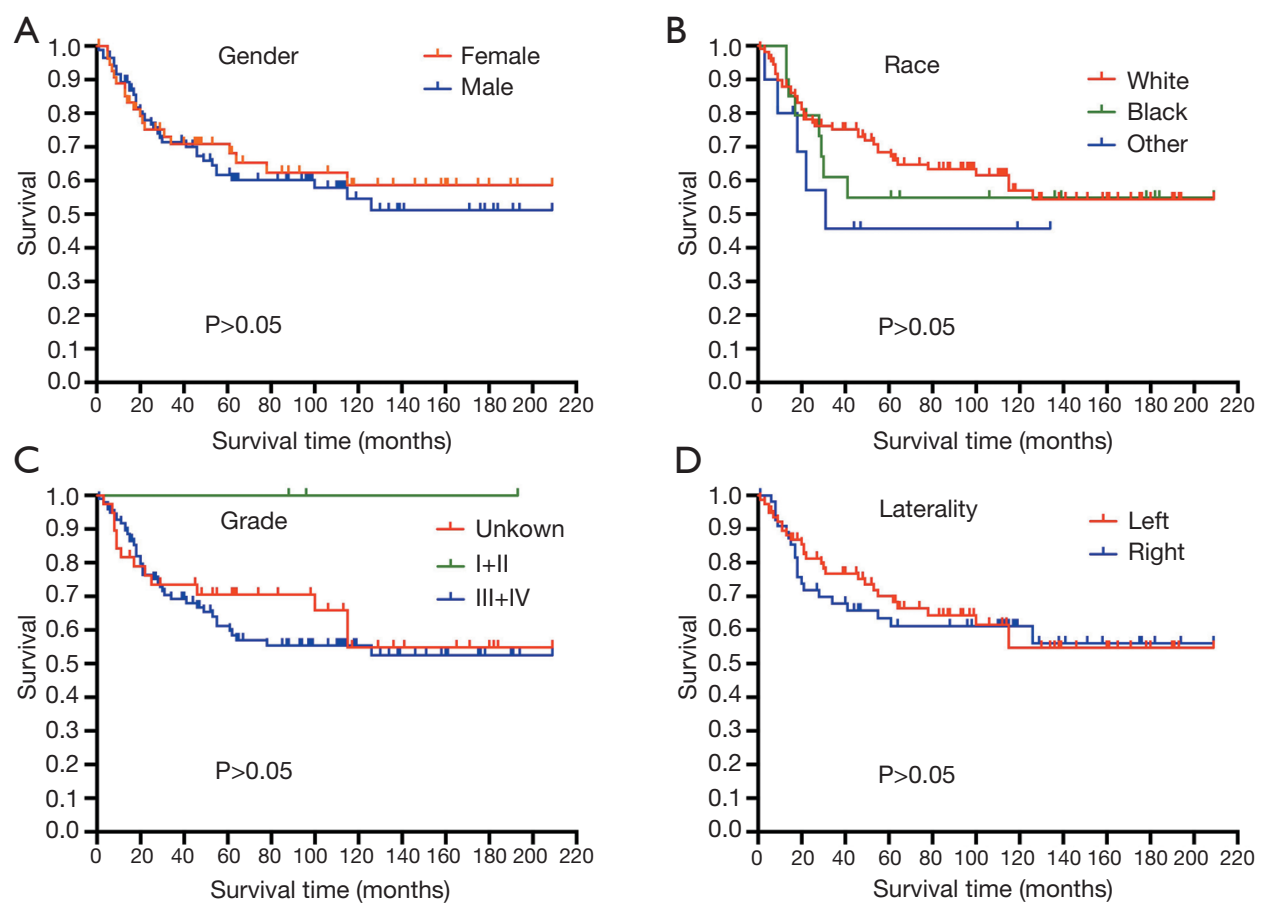

Figure 3 Kaplan-Meier curves revealed that TOS patient survival did not differ significantly as a function of gender, race, grade, laterality, or summary stage $(\mathrm{P}>0.05)$. TOS, telangiectatic osteosarcoma.

covariates in a subsequent multivariate analysis, which revealed that patients less than 20 years old had significantly better outcomes, and this was consistent with our univariate analysis. In addition, the multivariate analysis revealed that localized or regional disease and having undergone surgery were independently associated with the prognosis of TOS patients (HR 0.53; 95\% CI: 0.24 to $1.17 ; \mathrm{P}=0.116$ ).

\section{Discussion}

TOS is a form of high-grade osteosarcoma that accounts for approximately $6.8 \%$ of all such osteosarcoma cases $(6,20)$. In line with these prior reports, we found that TOS accounted for $3.1 \%$ of osteosarcoma cases in the SEER database. No large-scale clinical studies of TOS have been conducted to date, and as such animal studies and case reports are the 

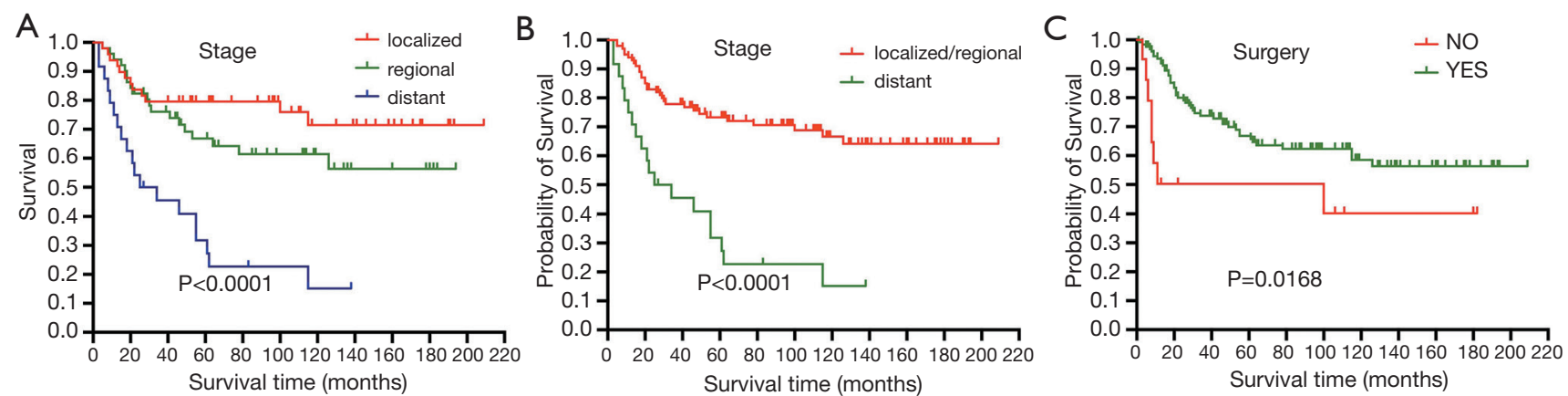

Figure 4 Kaplan-Meier survival curves of TOS patients according to tumor stage and surgery. (A,B) Kaplan-Meier curves revealed that earlystage disease was associated with better prognosis in TOS patients $(\mathrm{P}<0.0001)$; (C) Kaplan-Meier curves showed that TOS patients who underwent surgery at the primary site survived for significantly longer than patients who did not undergo surgery (not reached $v s .100$ months, HR 0.27, $95 \%$ CI: 0.09 to $0.79, \mathrm{P}<0.05)$. TOS, telangiectatic osteosarcoma; HR, hazard ratio; CI, confidence interval.

Table 2 The 5 - and 10-year survival rates of TOS patients

\begin{tabular}{lll}
\hline Age & 5 -year & 10 -year \\
\hline Overall population & $48.2 \%(68 / 141)$ & $20.6 \%(29 / 141)$ \\
$<20$ & $59.8 \%(52 / 87)$ & $21.8 \%(19 / 87)$ \\
$20-39$ & $36.7 \%(11 / 30)$ & $23.3 \%(7 / 30)$ \\
$40-59$ & $30.8 \%(4 / 13)$ & $23.1 \%(3 / 13)$ \\
$\geq 60$ & $9.1 \%(1 / 11)$ & $0 \%(0 / 11)$ \\
\hline
\end{tabular}

TOS, telangiectatic osteosarcoma.

primary sources of information pertaining to this disease. To better understand the characteristics of this condition, a comprehensive analysis of TOS cases in the SEER database was conducted.

In previous studies, TOS patients have been shown to exhibit symptoms, lesion locations, and demographics consistent with those of COS patients (21). In agreement with these findings, no significant differences were detected in gender, race, year of diagnosis, grade, or summary stage when comparing the TOS and COS patient cohorts in the SEER database. Consistent with our findings, osteosarcoma primarily affects children and young adults $(7,22)$. Indeed, the relative prevalence of TOS among persons under 20 years of age was even higher than that of COS (61.7\% vs. 51.7\%). In addition, TOS patients had a lower mean age compared to COS patients (23.5 vs. 29 years). Unlike COS, a second peak of incidence was not detected for TOS patients over the age of 60 years. The male-female ratio in this study was 1.2:1, which contrasts with prior investigations reporting that this disease is more commonly detected in males with a male- female ratio of 2.1:1 (7).

As relatively few TOS cases have been reported to date, the prognosis of this condition remains poorly understood. In a previous study, Matsuno et al. demonstrated that TOS patients had a poorer prognosis compared to COS patients (14), whereas other researchers have observed no differences in survival between these two disease types, and in fact, some reports have even found that TOS showed a mildly improved prognosis relative to conventional high-grade intramedullary osteosarcoma $(15,16)$. We consider that the limited number of cases account for the contradictory results. In line with these latter findings, we determined that TOS was associated with a significantly longer mOS compared to COS (not reached vs. 84 months; HR $0.75,95 \%$ CI: 0.59 to $0.95, \mathrm{P}=0.0175$ ), although this difference was not significant once it had been adjusted for age, stage, or surgical treatment. The small sample sizes of TOS might involve in the causes of no significant differences between two groups.

Studies in the literature (23-26) have demonstrated that age at diagnosis, tumor grade, and stage are associated with the prognosis of COS. Consistent with these results, we found that TOS patients under that age of 20 had a better prognosis, with the best prognosis being observed in patients under the age of 10 years. However, no significant differences in the prognosis of TOS patients over the age of 20 were observed.

The primary treatment for osteosarcoma is surgical resection. Consistent with this, TOS patients without metastatic disease at the time of diagnosis and patients who underwent surgical treatment were shown to have a better prognosis than patients with metastatic disease and patients 
Table 3 Univariate and multivariate analyses of the median overall survival of TOS patients

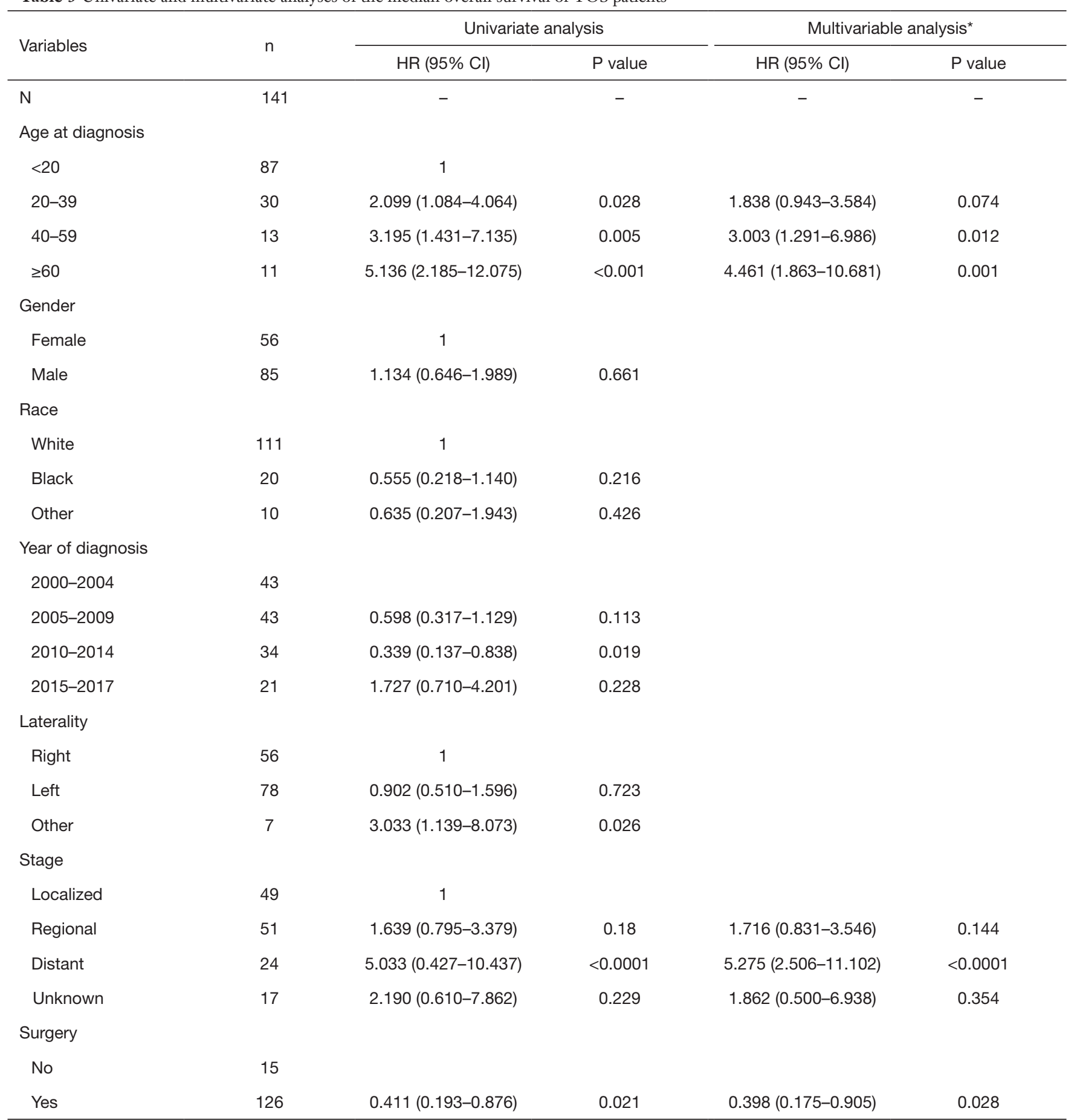

*, adjusted for age at diagnosis, summary stage, and surgery at primary site. TOS, telangiectatic osteosarcoma; HR, hazard ratio; Cl, confidence interval. 
who did not undergo surgery.

Through univariate and multivariate analyses, age at diagnosis, stage, and surgery at the primary site were all determined to be independent predictors of TOS patient survival. Patients less than 20 years old, patients with localized or regional disease, and patients who underwent surgery showed the best prognosis. As there were no significant differences in tumor stage distributions in the TOS and COS patient cohorts, we speculated that a younger age and higher surgical resection rates were related to the improved prognosis of TOS patients in this cohort. TOS patients who underwent surgery showed improved mOS, suggesting that surgical resection is an effective treatment for this type of osteosarcoma.

No significant differences were observed in the mOS of TOS patients as a function of patient gender, race, or laterality. Unexpectedly, TOS grade was not associated with patient prognosis. However, this may be a consequence of the fact that grade data were unavailable for many patients, with only three patients in the TOS cohort having been diagnosed with grade I/II disease.

It is important to differentiate between TOS and aneurysmal bone cysts, given that they exhibit similar radiological and pathological phenotypes $(27,28)$. Invasive growth pattern is occasionally accompanied by matrix mineralization, multiple fluid filled cavities of different sizes, surrounded by thick peripheral, septal and nodular tissue, which are helpful for the correct diagnosis of TOS (29). TOS lesions exhibit characteristic imaging findings that can guide the accurate preoperative diagnosis thereof including infiltrative growth, intermittent matrix mineralization, thickening of the peripheral tissue, the presence of multiple irregularly sized fluid-filled cavities, and the presence of septal tissue with surrounding nodule patterns. However, a pathological diagnosis is the gold standard and is necessary to formally confirm a diagnosis of TOS.

Through this study, we can have a better understanding of clinical characteristics and diagnosis of TOS. We will also further test the patient's gene and PD-L1 by Nextgeneration sequencing technology to further understand the disease and explore more advanced treatment option, such as target therapy and immune checkpoint inhibitors.

This study has several limitations. First, being a retrospective study, it was thus susceptible to selection bias. In addition, as the SEER database does not include gene expression data, we were not able to assess the relationship between gene expression patterns and patient survival outcomes. This database also does not include details pertaining to patient comorbidities, drug usage, surgical treatments, or radiotherapy doses, and therefore we were not able to evaluate the relationship between these parameters and patient outcomes. Time- and site-specific differences in data entry into this SEER database may also contribute to significant heterogeneity among these results. Despite these limitations, this study offers new insights that will help to guide future research pertaining to this rare disease.

In conclusion, in the present population-based study we evaluated and summarized the clinical characteristics of TOS to improve current awareness and diagnosis of this disease. TOS was most often detected in patients under 20 years of age, without a second peak of incidence after 60 years of age. There was a slightly higher incidence in males compared to females. Age at diagnosis, summary stage, and surgery at the primary site were identified as independent predictors of prognosis in TOS patients. The mOS of TOS patients was longer than that of COS patients, which may be due to the younger age of the patients and the higher surgical resection rate in TOS patients. The latter suggested that surgical resection is an effective treatment for patients with this disease.

\section{Acknowledgments}

Funding: This work was supported by grants from the Natural Science Foundation of Chpongqing, China No. cstc2020jcyj-msxmX0233 to MX, and MN was supported by the Kuanren Talents Program of the Second Affiliated Hospital of Chongqing Medical University.

\section{Footnote}

Reporting Checklist: The authors have completed the STROBE reporting checklist. Available at http://dx.doi. org/10.21037/atm-20-8001

Conflicts of Interest: All authors have completed the ICMJE uniform disclosure form (available at http://dx.doi. org/10.21037/atm-20-8001). The authors have no conflicts of interest to declare.

Ethical Statement: The authors are accountable for all aspects of the work in ensuring that questions related to the accuracy or integrity of any part of the work are appropriately investigated and resolved. The study was conducted in accordance with the Declaration of Helsinki (as 
revised in 2013).

Open Access Statement: This is an Open Access article distributed in accordance with the Creative Commons Attribution-NonCommercial-NoDerivs 4.0 International License (CC BY-NC-ND 4.0), which permits the noncommercial replication and distribution of the article with the strict proviso that no changes or edits are made and the original work is properly cited (including links to both the formal publication through the relevant DOI and the license). See: https://creativecommons.org/licenses/by-nc-nd/4.0/.

\section{References}

1. Valery PC, Laversanne M, Bray F. Bone cancer incidence by morphological subtype: a global assessment. Cancer Causes Control 2015;26:1127-39.

2. Green JT, Mills AM. Osteogenic tumors of bone. Seminars in Diagnostic Pathology 2014;31:21-9.

3. Yarmish G, Klein MJ, Landa J, et al. Imaging characteristics of primary osteosarcoma: nonconventional subtypes. Radiographics 2010;30:1653-72.

4. Gennari L, Rendina D, Falchetti A, et al. Paget's Disease of Bone. Calcif Tissue Int 2019;104:483-500.

5. Sangle NA, Layfield LJ. Telangiectatic osteosarcoma. Arch Pathol Lab Med 2012;136:572-6.

6. Limaiem F, Kuhn J, Khaddour K. Cancer, Telangiectatic Osteosarcoma. StatPearls. Treasure Island (FL) 2020. Available online: https://www.ncbi.nlm.nih.gov/books/ NBK537309/

7. Murphey MD, wan Jaovisidha S, Temple HT, et al. Telangiectatic osteosarcoma: radiologic-pathologic comparison. Radiology 2003;229:545-53.

8. Tomar D, Dhillon M, Thayath MN, et al. Central Telangiectatic Osteosarcoma of the Mandible in a Paediatric Patient: A Rarity. J Clin Diagn Res 2016;10:XD01-XD03.

9. Colomina J, Peiro A, Trullols L, et al. Telangiectatic osteosarcoma. J Orthop Surg (Hong Kong) 2013;21:96-9.

10. Naik LK, Shetty P, Teerthanath S, et al. Telangiectatic osteosarcoma affecting the mandible. J Oral Maxillofac Pathol 2014;18:S143-6.

11. Giuffrida MA, Kamstock DA, Selmic LE, et al. Primary appendicular hemangiosarcoma and telangiectatic osteosarcoma in 70 dogs: A Veterinary Society of Surgical Oncology retrospective study. Vet Surg 2018;47:774-83.

12. Goldschmidt B, Calado MI, Resende FC, et al. Spontaneous telangiectatic osteosarcoma in a rhesus macaque (Macaca mulatta). J Med Primatol 2017;46:51-5.

13. Giuffrida MA, Bacon NJ, Kamstock DA. Use of routine histopathology and factor VIII-related antigen/von Willebrand factor immunohistochemistry to differentiate primary hemangiosarcoma of bone from telangiectatic osteosarcoma in 54 dogs. Vet Comp Oncol 2017;15:1232-9.

14. Matsuno T, Unni KK, McLeod RA, et al. Telangiectatic osteogenic sarcoma. Cancer 1976;38:2538-47.

15. Bacci G, Picci P, Ferrari S, et al. Primary chemotherapy and delayed surgery for non-metastatic telangiectatic osteosarcoma of the extremities. Results in 28 patients. Eur J Cancer 1994;30A:620-6.

16. Pignatti G, Bacci G, Picci P, et al. Telangiectatic osteogenic sarcoma of the extremities. Results in 17 patients treated with neoadjuvant chemotherapy. Clin Orthop Relat Res 1991:99-106.

17. Xu Z, Wang L, Tu L, et al. Epidemiology of and prognostic factors for patients with sarcomatoid carcinoma: a large population-based study. Am J Cancer Res 2020;10:3801-3814.

18. Xu S, Li X, Ren F, et al. Sublobar Resection Versus Lobectomy for Early-Stage Pulmonary Carcinoid Tumors $\leq 3 \mathrm{~cm}$ in Size: A SEER Population-Based Study. Ann Surg 2020. [Epub ahead of print]. doi: 10.1097/ SLA.0000000000004593.

19. Wagner MJ, Chau B, Loggers ET, et al. Long-term Outcomes for Extraskeletal Myxoid Chondrosarcoma: A SEER Database Analysis. Cancer Epidemiol Biomarkers Prev 2020;29:2351-7.

20. Weiss A, Khoury JD, Hoffer FA, et al. Telangiectatic osteosarcoma: the St. Jude Children's Research Hospital's experience. Cancer 2007;109:1627-37.

21. Murphey MD, Robbin MR, McRae GA, et al. The many faces of osteosarcoma. Radiographics 1997;17:1205-31.

22. Ottaviani G, Jaffe N. The epidemiology of osteosarcoma. Cancer Treat Res 2009;152:3-13.

23. Duchman KR, Gao Y, Miller BJ. Prognostic factors for survival in patients with high-grade osteosarcoma using the Surveillance, Epidemiology, and End Results (SEER) Program database. Cancer Epidemiol 2015;39:593-9.

24. Schlegel M, Zeumer M, Prodinger PM, et al. Impact of Pathological Fractures on the Prognosis of Primary Malignant Bone Sarcoma in Children and Adults: A Single-Center Retrospective Study of 205 Patients. Oncology 2018;94:354-62.

25. Anderson ME. Update on survival in osteosarcoma. Orthop Clin North Am 2016;47:283-92.

26. Song K, Song J, Chen F, et al. Prognostic nomograms for 
predicting overall and cancer-specific survival of high-grade osteosarcoma patients. J Bone Oncol 2018;13:106-13.

27. Zishan US, Pressney I, Khoo M, et al. The differentiation between aneurysmal bone cyst and telangiectatic osteosarcoma: a clinical, radiographic and MRI study. Skeletal Radiol 2020;49:1375-86.

28. Yin JQ, Fu YW, Xie XB, et al. Telangiectatic osteosarcoma: Outcome analyses and a diagnostic model for

Cite this article as: $\mathrm{Xu} M$, Dai N, Yang X, Guan W, Pu Y, Wang D, Yin L, Nie M. Characteristics and prognosis of telangiectatic osteosarcoma: a population-based study using the Surveillance, Epidemiology and End Results (SEER) database. Ann Transl Med 2021;9(9):796. doi: 10.21037/atm-20-8001 differentiation from aneurysmal bone cyst. J Bone Oncol 2017;11:10-6.

29. Gao ZH, Yin JQ, Liu DW, et al. Preoperative easily misdiagnosed telangiectatic osteosarcoma: clinicalradiologic-pathologic correlations. Cancer Imaging 2013;13:520-6.

(English Language Editor: J. Teoh) 